\title{
Development of stacked core technology for the fabrication of deep lightweight UV quality space mirrors
}

\author{
Gary Matthews, Charlie Kirk, Steve Maffett and Cal Abplanalp \\ Exelis Inc. \\ H. Philip Stahl \\ NASA Marshall Space Flight Center \\ SUBMITTED to: \\ SPIE Conference on \\ Optical Manufacturing and Testing X \\ San Diego CA \\ August 25-29, 2013
}

\begin{abstract}
Decadal Survey stated that an advanced large-aperture ultraviolet, optical, near-infrared (UVOIR) telescope is required to enable the next generation of compelling astrophysics and exoplanet science; and, that present technology is not mature enough to affordably build and launch any potential UVOIR mission concept. Under Science and Technology funding, NASA's Marshall Space Flight Center (MSFC) and ITT Exelis have developed a more cost effective process to make up to $4 \mathrm{~m}$ monolithic spaceflight UV quality, low areal density, thermally and dynamically stable primary mirrors. A proof of concept mirror was completed at ITT Exelis and tested down to $250 \mathrm{~K}$ at MSFC which would allow imaging out to 2.5 microns. The parameters and test results of this concept mirror will be shown. The scale-up process will be discussed and the technology development path to a $4 \mathrm{~m}$ mirror system by 2018 will also be outlined.
\end{abstract}

